\title{
VLBI observations of gamma-ray binaries: LS 5039 and PSR B1259-63
}

\author{
Javier Moldón*, Marc Ribó, Josep M. Paredes \\ Departament d'Astronomia i Meteorologia, Institut de Ciències del Cosmos (ICC), Universitat \\ de Barcelona (IEEC-UB) \\ Martí i Franquès, 1 \\ 08028 Barcelona, Spain \\ E-mail: jmoldon@am.ub.es, mribodam.ub.es, jmparedes@ub.edu

\section{W. Brisken} \\ National Radio Astronomy Observatory \\ E-mail: wbriskendaoc.nrao.edu

\section{Kramer} \\ Jodrell Bank Centre for Astrophysics \\ Max Planck Institut für Radioastronomie \\ E-mail: mkramer@mpifr-bonn.mpg •de
}

\section{A.G. Lyne}

Jodrell Bank Centre for Astrophysics

E-mail: andrew. lyne@manchester.ac.uk

\section{B.W. Stappers}

Jodrell Bank Centre for Astrophysics

E-mail: ben.stappers@manchester.ac.uk

\section{Simon Johnston}

Australia Telescope National Facility, CSIRO

E-mail: Simon.Johnston@atnf.csiro.au

\section{Adam Deller}

National Radio Astronomy Observatory

Astronomy Department, University of California at Berkeley

E-mail: adellerenrao.edu 
$\mathrm{X}$-ray binaries that have been unambiguously detected at $\mathrm{TeV}$ energies show a peculiar radio morphological variability at AU scales, which can be used to gain knowledge on the particle accelerator that powers their broadband emission. We present a multi-epoch VLBI study of LS 5039, including Very Long Baseline Array (VLBA) observations at $5 \mathrm{GHz}$ covering an orbital cycle. We discuss the possible relation of LS 5039 and the pulsar PSR J1825-1446 with the nearby supernova remnant SNR G016.8-01.1 by means of proper motion studies. Our preliminary results indicate either that we are facing with a very old SNR related to LS 5039 or that it is a relatively young SNR related to PSR J1825-1446, in which case the kinematical age of this pulsar would be one order of magnitude smaller than its characteristic age. In addition, we present observations of PSR B1259-63 obtained with the Australian Long Baseline Array (LBA) at 2.3 GHz during the 2007 periastron passage. We have discovered extended and variable radio structure. The peak of the radio emission is detected outside the binary system near periastron, and the emission has a total extent of $120 \mathrm{AU}$. This is the first observational evidence that non-accreting pulsars orbiting massive stars can produce variable extended radio emission at AU scales. The discovery presented here for the young non-accreting pulsar PSR B1259-63 reinforces the link with the other two known gamma-ray binaries, namely LS 5039 and LS I +61 303, and supports the presence of pulsars in these systems as well.

10th European VLBI Network Symposium and EVN Users Meeting: VLBI and the new generation of radio arrays

September 20-24, 2010

Manchester Uk

${ }^{*}$ Speaker. 


\section{Introduction}

LS 5039, PSR B1259-63 and LS I+61 303 are high mass binaries that display high-energy (HE) and very high energy (VHE) gamma-ray emission $(E>100 \mathrm{GeV})$ with a clear orbital variability [3, 2, 4, 5]. The HE/VHE emission is basically interpreted as the result of inverse Compton upscattering of stellar UV photons by relativistic electrons. The acceleration of electrons seems to be produced by shocks between the relativistic wind of a young non-accreting pulsar and the wind of the stellar companion [10, 17]. The three sources show a very similar broadband spectral energy distribution (SED) [10]. Contrary to X-ray binaries, all three sources have the peak of the SED at $\mathrm{MeV}-\mathrm{GeV}$ energies. For these reasons, they can be considered gamma-ray binaries. However, the nature of the compact objects in LS 5039 and LS I+61 303 is unknown because their masses are not well constrained by the system mass functions [8, 9, 7], and no pulsations have been found. These systems have been extensively observed at VHE during several orbital cycles, while the observations of PSR B1259-63, the only system with a confirmed pulsar, are scarce due to the long orbital period of $3.4 \mathrm{yr}$. Any observational link between the three gamma-ray binaries would shed light in the understanding of this kind of systems.

On the other hand, Cygnus X-3 was detected above $100 \mathrm{MeV}$ by the AGILE and Fermi satellites [18, 1]. The MAGIC collaboration reported evidence of a short flare of 80 minutes from Cygnus X-1 at TeV energies [6], and Sabatini et al. (2010) [16] reported a flare with AGILE. However, the nature of these detections, flares not correlated with the orbital phase but probably with the spectral accretion state, seems different from the observed behavior in the gamma-ray binaries.

In all the proposed models for gamma-ray binaries the multiwavelength emission depends on the geometry of the region where particles are accelerated, and its location with respect to the observer. In this context, VLBI radio observations can provide a direct view of the small scale structure of these systems, which, at radio wavelengths, is expected to be observable at AU scales. LS 5039 is located at $2.5 \mathrm{kpc}$ [9], and PSR B1259-63 at $2.3 \mathrm{kpc}$ [11] and hence if we study the source morphology at 1-100 mas scales we are sampling projected distances around 2.5-250 AU.

\section{VLBA observations of LS 5039}

LS 5039 has been observed with the VLBA at $5 \mathrm{GHz}$, showing bipolar extended emission of a few mJy extending over 6 mas on the plane of the sky [13], which is variable in orbital timescales[15]: the Position Angle (P.A.) of the direction of the elongated emission changed by $12 \pm 3^{\circ}$ between runs separated 5 days. To further study the morphological variability, we observed the source in 2007 during five consecutive days. The self-calibrated images show a main core and extended emission up to $\sim 6$ mas, as in previous observations. The P.A. of the extended emission from the main core changes significantly every day, covering P.A. between -50 and $-85^{\circ}$ for orbital phases in the range $0.5-0.0$, and P.A. $120^{\circ}$ at orbital phase 0.23 . Therefore, a subtle change in morphology happens in less than 20 hours after periastron.

As a by-product, these phase-referenced observations provide one average precise position of the source in the sky. Ribó et al. (2002) [14] computed the trajectory of LS 5039 for the last $10^{5} \mathrm{yr}$ using optical/radio astrometry from 1905 to 2002. Their result marginally suggests an association with SNR G016.8-01.1. This new VLBA position, combined with previous radio interferometric 
measurements spanning 9 years, allow us to calculate a more precise proper motion and past trajectory of the source (see solid line in Fig. 1). Our updated past trajectory of LS 5039 is compatible with the center of the SNR. With a firm association, a kinematical age of the compact object would be obtained, which would have direct implications on the binary properties. In case the system contains a pulsar, the kinematic age indicates that it would be young enough to be in the non-accreting phase, and therefore, compatible with the shocked winds scenario. On the other hand, we started an astrometric project to measure the proper motion of a nearby pulsar, PSR J1825-1446. After the first two epochs we have obtained a proper motion of $\sim 30$ mas yr$^{-1}$, which corresponds to a $2 \mathrm{D}$ projected velocity of $780 \mathrm{~km} \mathrm{~s}^{-1}$ at $5.5 \mathrm{kpc}$. This means that this pulsar may be a high speed pulsar ejected from the Galactic plane. The past trajectory of PSR J1825-1446 is also compatible with the center of the SNR within errors. In case the pulsar was formed in that supernova explosion, its kinematical age would be around $10000 \mathrm{yr}$. The characteristic age of this pulsar is one order of magnitude bigger than that age, although it is known that the characteristic ages of pulsars are usually an overestimation of the real age. Further epochs of the astrometric project will better restrict the proper motion of PSR J1825-1446, and will allow us to better understand the possible relations of SNR G016.8-01.1 with LS 5039 and PSR J1825-1446.

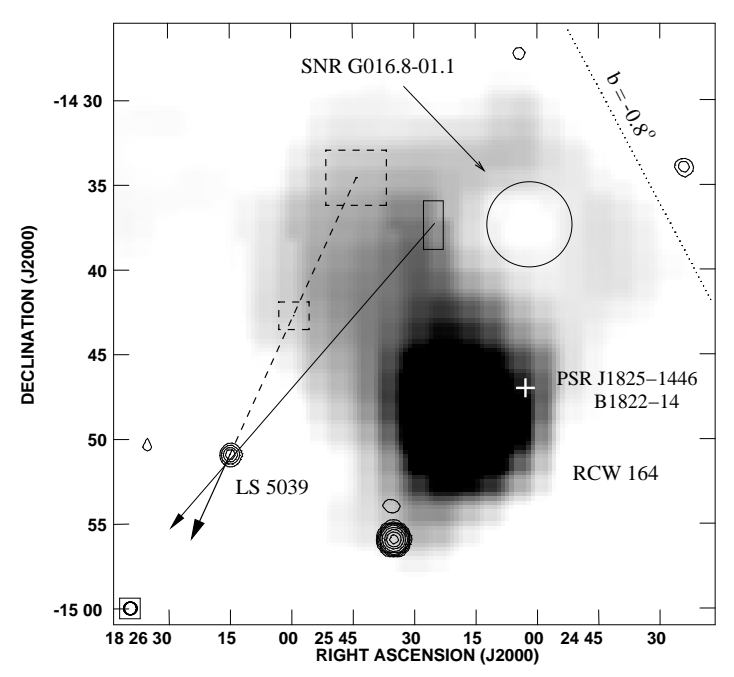

Figure 1: Wide field radio map of LS 5039, showing the position of the nearby SNR G016.8-01.1. The grey scale emission is at the $6 \mathrm{~cm}$ wavelength (obtained with single dish). The overlaid contours correspond to the NVSS map at $20 \mathrm{~cm}$. The arrows mark the proper motion senses. The dashed line is the computed trajectory reported in Ribó et al. (2002), whereas the solid line is the computed trajectory from our fit (see text). The error boxes are calculated for $5 \times 10^{4}$ and $10^{5} \mathrm{yr}$ ago for the Ribó et al. (2002) fit, and $10^{5} \mathrm{yr}$ ago for our updated fit.

\section{LBA observations of PSR B1259-63}

PSR B1259-63 was observed with the Australian Long Baseline Array (LBA) at $2.3 \mathrm{GHz}$ $(13 \mathrm{~cm}$ ) on three epochs: 2007 July 28 (run A), 2007 August 17 (run B), and 2008 June 6 (run C), see [12]. The resulting VLBI images at $2.3 \mathrm{GHz}$ are shown in Figure 2. Extended emission is 
detected at distances up to 50-55 mas (120-130 $\pm 20 \mathrm{AU}$ at $2.3 \pm 0.4 \mathrm{kpc})$ during the two runs shortly after the periastron passage. The Position Angle (P.A.) of the extended emission with respect to the peak is $\sim-67^{\circ}$ for run $\mathrm{A}$ and $\sim-50^{\circ}$ for run $\mathrm{B}$. In these runs, the peak of the emission is clearly displaced from the binary system orbit. The emission in run C, 315 days after the periastron passage, is dominated by a point-like source of a few mJy. As a reference position for the plots we use the peak position of run $\mathrm{C}$, which we consider to represent the pulsar position (see Moldón et al, submitted to ApJ [12]).

We confirm that non-accreting pulsars orbiting massive stars can produce variable extended radio emission at $\mathrm{AU}$ scales. The P.A. of the extended emission with respect to the brightest point of the image changes $\sim 10-20^{\circ}$ in 20 days. Similar morphological changes have been found in the other two known gamma-ray binaries, LS 5039 and LS I +61 303, although for smaller sizes and on shorter timescales.
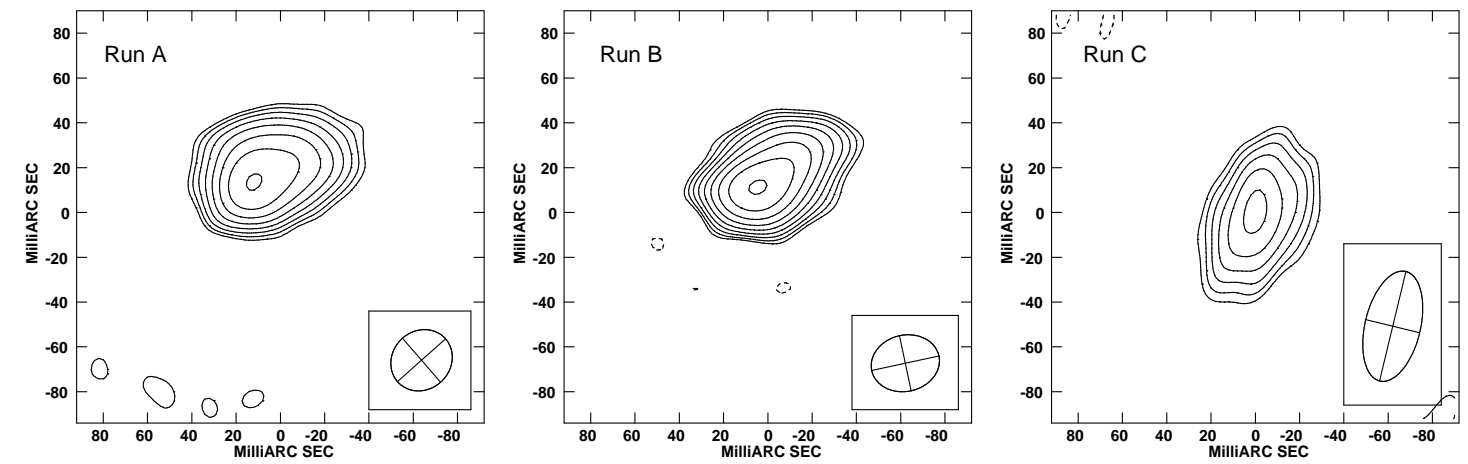

Figure 2: LBA images of PSR B1259-63 at $2.3 \mathrm{GHz}$. North is up and East is to the left. The synthesized beam is displayed in the rectangle on the bottom-right corner of each image. For each image, the displayed contours start at $3 \sigma$ and increase by factors of $2^{1 / 2}$; the $1-\sigma$ rms close to the source in each image from left to right is $0.30,0.66$, and $0.15 \mathrm{mJy}_{\text {beam }}^{-1}$.

\section{Discussion}

The small scale structure of gamma-ray binaries can be studied by means of VLBI radio observations, which provide a direct view of the morphology of the extended emission at AU scales, while they provide accurate astrometry useful for obtaining proper motions, which can help to identify the origin of these systems. We have observed LS 5039 and PSR B1259-63 at different orbital phases. In both cases, we have seen variable extended emission. The radio morphology at mas scales of LS 5039 shows changes in $24 \mathrm{~h}$, and there is orbital morphological variability. However, new VLBI observations are required at higher frequencies to accurately trace the core motion along the orbit. We have also seen morphological changes in PSR B1259-63 at two epochs separated 20 days near the periastron passage. The total projected extent of the nebula is $\sim 50$ mas, or $120 \pm 20 \mathrm{AU}$, and the peak of the emission is clearly displaced from the binary system orbit. The discovery of such a structure in PSR B1259-63 reinforces the link with the other known gammaray binaries, LS 5039 and LS I +61 303, for which the detection of pulsations is challenging. 
On the other hand, proper motion measurements of LS 5039 suggest that the compact object of this gamma-ray binary could have been produced in the same supernova explosion as SNR G016.8-01.1. If this is the case, the compact object in LS 5039 would be $10^{5} \mathrm{yr}$ old. However, PSR J1825-1446 also appears to come from SNR G016.8-01.1. If this is the case, the characteristic age would be one order of magnitude higher than the kinematical age.

\section{References}

[1] Abdo, A. A., et al. 2009a, Science, 326, 1512

[2] Abdo, A. A., et al. 2009b, ApJL, 706, L56

[3] Aharonian, F. et al. 2006, A\&A, 460, 743

[4] Aharonian, F., Akhperjanian, A. G., Anton, G., et al. 2009, A\&A, 507, 389

[5] Albert, J., Aliu, E., Anderhub, H., et al. 2006, Science, 312, 1771

[6] Albert, J., et al. 2007, ApJL, 665, L51

[7] Aragona, C., McSwain, M. V., Grundstrom, E. D., Marsh, A. N., Roettenbacher, R. M., Hessler, K. M., Boyajian, T. S., \& Ray, P. S. 2009, ApJ, 698, 514

[8] Casares, J., Ribas, I., Paredes, J. M., Martí, J., \& Allende Prieto, C. 2005a, MNRAS, 360, 1105

[9] Casares, J., Ribó, M., Ribas, I., Paredes, J. M., Martí, J., \& Herrero, A. 2005b, MNRAS, 364, 899

[10] Dubus, G. 2006, A\&A, 456, 801

[11] Negueruela, I., Ribó, M., Herrero, A., Lorenzo, J., Khangulyan, D., \& Aharonian, F. A. 2010, ApJL, submitted

[12] Moldón, J., Johnston, S., Ribó, M., Paredes, J. M., \& Deller, A. 2010, ApJL, submitted

[13] Paredes, J. M., Martí, J., Ribó, M., \& Massi, M. 2000, Science, 288, 2340

[14] Ribó, M., Paredes, J. M., Romero, G. E. et al. 2002, A\&A 384, 954

[15] Ribó, M., Paredes, J. M., Moldón, J. Martí, J., \& Massi, M. 2008, A\&A, 481, 17

[16] Sabatini, S., et al. 2010, ApJL, 712, L10

[17] Sierpowska-Bartosik, A., \& Torres, D. F. 2008, Astroparticle Physics, 30, 239

[18] Tavani, M., et al. 2009, Nature, 462, 620 